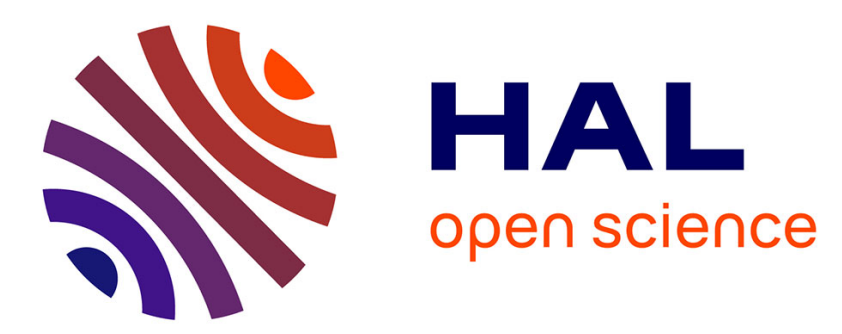

\title{
On modeling shape memory polymers as elastic two-phase composite materials
}

Pierre Gilormini, Julie Diani

\section{To cite this version:}

Pierre Gilormini, Julie Diani. On modeling shape memory polymers as elastic two-phase composite materials. Comptes Rendus Mécanique, 2012, 340 (4-5), p. 338-348. 10.1016/j.crme.2012.02.016 . hal-00755425

\section{HAL Id: hal-00755425 \\ https://hal.science/hal-00755425}

Submitted on 21 Nov 2012

HAL is a multi-disciplinary open access archive for the deposit and dissemination of scientific research documents, whether they are published or not. The documents may come from teaching and research institutions in France or abroad, or from public or private research centers.
L'archive ouverte pluridisciplinaire HAL, est destinée au dépôt et à la diffusion de documents scientifiques de niveau recherche, publiés ou non, émanant des établissements d'enseignement et de recherche français ou étrangers, des laboratoires publics ou privés. 


\title{
On modeling shape memory polymers as thermoelastic two-phase composite materials
}

\author{
P. Gilormini, J. Diani \\ Laboratoire Procédés et Ingénierie en Mécanique et Matériaux, \\ Arts et Métiers ParisTech, CNRS, 151 Bd de l'Hopital, 75013 Paris, France
}

\begin{abstract}
A model has been proposed recently, which describes the experimentally observed mechanical behavior of some shape memory polymers. It considers a purely thermoelastic behavior, without strain rate effects, and assumes essentially that the polymer can be considered as a two-phase composite, with glassy and rubbery phases having volume fractions that depend on temperature only. Since a uniform stress hypothesis was used in the original formulation, with an inconsistency when thermal expansion was considered, this model is revisited here by taking advantage of the many results that have been established in the theory of composite materials. It is shown, especially, that a uniform strain hypothesis is more appropriate than assuming a uniform stress. To cite this article: P. Gilormini, J. Diani, C. R. Mecanique xxx (2011).
\end{abstract}

\section{Résumé}

Sur la modélisation des polymères à mémoire de forme comme des composites thermoélastiques biphasés. Un modèle proposé récemment décrit le comportement mécanique observé sur des polymères à mémoire de forme. Il considère un comportement purement thermoélastique, sans effets visqueux, et fait principalement l'hypothèse que le matériau peut être considéré comme un composite à deux phases, l'une vitreuse et l'autre caoutchoutique, dans des proportions qui dépendent de la seule température. Comme une hypothèse de contraintes uniformes était utilisée, de façon non cohérente avec l'évaluation faite de la dilatation thermique effective, ce modèle est révisé ici en exploitant en particulier les nombreux résultats connus en mécanique des composites. On montre en particulier que supposer une déformation uniforme serait plus approprié. Pour citer cet article : P. Gilormini, J. Diani, C. R. Mecanique xxx (2011).

Key words: Shape memory; Polymers

Mots-clés : Mémoire de forme; Polymères

Email addresses: pierre.gilormini@ensam.eu (P. Gilormini), julie.diani@ensam.eu (J. Diani). 


\section{Introduction}

Shape memory polymers are smart materials for which interest is growing very rapidly. For instance, the literature search conducted by Liu et al. [1] showed an almost exponential increase of publications on this topic since 1980. Numerous applications are being explored, which require the development of new compositions and structures, with new challenges to be faced, as presented in such recent reviews as by Liu et al. [1], Rousseau [2] or Mather et al. [3].

A large part of these papers is devoted to the development of new shape memory polymers or to the measure of their properties, but much less work is done on modeling their mechanical behavior. The models that have been proposed so far for thermally actuated shape memory polymers, to which this paper is limited, are of two types according to whether rate effects are included or not. In the first type, Tobushi et al. [4] developed a four-element variation of a viscoelastic rheological model, the numerical integration of which was detailed by Bhattacharyya and Tobushi [5], and that was extended to nonlinear effects by Tobushi et al. [6], whereas Lin and Chen [7] could reproduce experimental results qualitatively with a mere 3-element standard linear solid by relating the dash-pot viscosity to temperature. A less macroscopic approach, using two coexisting substructures in the material, with different mechanical behaviors, was proposed by Kafka [8], while Qi et al. [9], Nguyen et al. [10], Srivastava et al. [11] developed other models by taking advantage of the large amount of knowledge that is available on polymer physics. All these models, as well as those considered below, apply to amorphous shape memory polymers and do not account for the specific effects of crystallization that Barot and Rao [12] and Barot et al. [13] included. The second type of models is simpler in essence, since rate effects are ignored. This can be considered as a first approximation of more elaborate models that may be appropriate for very low, or constant, strain rate and temperature rate. The paper by Liu et al. [14] is seminal in this respect: a purely thermoelastic two-phase model was proposed and a set of experimental results using a fixed temperature rate was presented, that have been the subject of several subsequent papers by various authors. An encouraging agreement was found with experiments, which confirmed that a thermoelastic model is able to capture the basic stress and strain evolutions. Extensions of the model to finite strain were proposed by Diani et al. [15] and by Chen and Lagoudas [16], who also provided a small-strain version (Chen and Lagoudas, [17]). The original small-strain model of Liu et al. [14] has also been revisited by Wang et al. [18], who proposed a first combination of the model with the theory of composite materials. Very recently, Reese et al. [19] developed their own finite strain version of a two-phase purely elastic model and implemented it in a finite element code in order to perform the simulation of the loading and expansion of stents with complex shapes. This illustrates a typical application of shape memory polymers today.

The present work revisits the thermoelastic two-phase model of Liu et al. [14], where glassy and rubbery phases have volume fractions that depend on temperature only, by using the theory of composite materials in a more systematic and consistent manner. The original model assumed a uniform stress distribution in the material, whereas several possibilities are considered here, which range from uniform stress to uniform strain. Moreover, the coefficient of thermal expansion is evaluated consistently with the mechanical behavior, which was not the case in [14]. Finally, the predictions of the various possibilities considered are compared with the experimental results reported in [14], and it is shown that assuming a uniform stress is not appropriate.

\section{Reformulation and extension of the previous model}

Like in [14], the polymer is described as a composite material where two phases coexist in the temperature range that define the glass transition, with volume fractions that depend on temperature only. The 
glassy (or "frozen") phase, with a volume fraction $\phi(T)$, has an elastic behavior that stems from internal energy, which arises from interatomic Van der Waals interactions and covalent intramolecular interactions, whereas the elastic behavior of the rubbery (or "active") phase, with a volume fraction $1-\phi(T)$, originates essentially from the entropy of the macromolecular chains. In a cooling process, for instance, glass transition develops from a high temperature $T_{+}$where (and above which) $\phi(T)=0$ to a low temperature $T_{-}$where (and below which) $\phi(T)=1$. It may be emphasized that what is meant here by "the glassy phase" is just the collection of all chains, or chain segments, in the polymer that have lost their mobility at a given temperature below $T_{+}$. This does not necessarily correspond to a continuous volume in the polymer, as would be the case in a composite with a continuous matrix, for instance, although the terms "composite" and "two-phase material" are used in this paper for the polymer. The $\phi(T)$ function plays a central role in the model, and was taken of the following empirical form by Liu et al. [14]

$$
\phi(T)=1-\frac{1}{1+c\left(T_{+}-T\right)^{n}}
$$

which does give $\phi\left(T_{+}\right)=0$ but not $\phi\left(T_{-}\right)=1$ exactly. This is why the following empirical relation, which generates similar sigmoidal-shaped curves,

$$
\phi(T)=\left[1-\left(\frac{T-T_{-}}{T_{+}-T_{-}}\right)^{m}\right]^{n}
$$

is prefered here, taking $\phi(T)=0$ if $T>T_{+}$and $\phi(T)=1$ if $T<T_{-}$. This also differs from the expression used by Wang et al. [18], with an exponential function and two variants for cooling and heating.

The model can be developed in three steps, which are detailed in the following three paragraphs and which all use the $\phi(T)$ function for evaluating the overall elastic behavior of the composite, defining its overall thermal expansion, and describing the shape memory effect.

\subsection{Overall elastic behavior}

In the theory of random composites, a set of models that apply to small-strain linear elasticity can be defined by using a comparison material, as explained by Willis [20] and as summarized by Zaoui [21]. In the present two-phase context, this leads to the following expression for the overall elastic behavior of the composite:

$$
\boldsymbol{L}=-\boldsymbol{L}^{\star}+\left[\phi\left(\boldsymbol{L}^{\star}+\boldsymbol{L}_{g}\right)^{-1}+(1-\phi)\left(\boldsymbol{L}^{\star}+\boldsymbol{L}_{r}\right)^{-1}\right]^{-1}
$$

where $\boldsymbol{L}_{g}$ and $\boldsymbol{L}_{r}$ denote the stiffness tensors of the glassy and rubbery phases, respectively, $\boldsymbol{L}^{\star}$ is the constraint tensor introduced by Hill [22] and pertaining to a comparison material with a stiffness $\boldsymbol{L}_{0}$ defined below. The phases are assumed to be isotropic, with bulk moduli $k_{g}$ and $k_{r}$ and shear moduli $\mu_{g}$ and $\mu_{r}$, with statistically isotropic distributions, so that the overall behavior also is isotropic, defined by $k$ and $\mu$. Therefore, the constraint tensor has the same structure as an isotropic stiffness tensor with a bulk modulus and a shear modulus given by

$$
k^{\star}=\frac{4}{3} \mu_{0} \quad \text { and } \quad \mu^{\star}=\frac{\mu_{0}}{6} \frac{9 k_{0}+8 \mu_{0}}{k_{0}+2 \mu_{0}}
$$

where $k_{0}$ and $\mu_{0}$ define the isotropic stiffness of the comparison material.

Isotropy of the phases and of the composite allows deducing the overall bulk modulus and shear modulus from (3) as

$$
k=k_{r}+\phi \frac{k_{g}-k_{r}}{1+(1-\phi) \frac{k_{g}-k_{r}}{k^{\star}+k_{r}}} \quad \text { and } \quad \mu=\mu_{r}+\phi \frac{\mu_{g}-\mu_{r}}{1+(1-\phi) \frac{\mu_{g}-\mu_{r}}{\mu^{\star}+\mu_{r}}}
$$

and the role of the comparison material is easily understood with these expressions. If $k_{0}=0$ and $\mu_{0}=0$, for instance, (5) leads to $1 / k=\phi / k_{g}+(1-\phi) / k_{r}$ and $1 / \mu=\phi / \mu_{g}+(1-\phi) / \mu_{r}$, i.e. to the uniform stress 
Reuss model, which gives lower bounds for $k$ and $\mu$, whereas letting $k_{0}$ and $\mu_{0}$ tend to infinity leads to $k=\phi k_{g}+(1-\phi) k_{r}$ and $\mu=\phi \mu_{g}+(1-\phi) \mu_{r}$, i.e. to the uniform strain Voigt model, which gives upper bounds. Since the moduli of the rubbery phase are lower than the moduli of the glassy phase, taking $k_{0}=k_{r}$ and $\mu_{0}=\mu_{r}$ transforms (3) into the expression of the Hashin and Shtrikman [23] lower bound, and using $k_{0}=k_{g}$ and $\mu_{0}=\mu_{g}$ gives the Hashin and Shtrikman upper bound. These bounds also coincide with the Mori and Tanaka [24] model for random distributions of spherical glassy inclusions in a rubbery matrix and of rubbery inclusions in a glassy matrix, respectively, especially when the reformulation given by Benveniste [25] is used. Finally, writing $k_{0}=k$ and $\mu_{0}=\mu$ transforms (3) into a coupled set of implicit equations with respect to $k$ and $\mu$ that corresponds to the standard self-consistent model as defined by Hill [22].

Therefore, a single set of simple scalar equations, (4) and (3), is used in this work to cover various standard models and bounds: the Reuss and Voigt bounds, the tighter Hashin and Strikman bounds, which do apply in the present context of a statistically isotropic distribution of two isotropic phases, the Mori and Tanaka model, with either a rubbery or a glassy continuous matrix, and the self-consistent model. This extends significantly the scope of the original model of Liu et al. [14], where the Reuss bound only was used.

\subsection{Overall thermal expansion}

Similarly to what has been done for their elastic behaviors, the thermal expansions of the two phases are assumed to be isotropic, defined by coefficients $\alpha_{g}$ and $\alpha_{r}$, and the overall thermal expansion also is isotropic, with a coefficient $\alpha$. In these conditions, the relation derived by Levin [26] and generalized by Rosen and Hashin [27] can be used to obtain $\alpha$ directly from the overall bulk modulus of the composite:

$$
\alpha=\phi \alpha_{g}+(1-\phi) \alpha_{r}+\frac{\frac{1}{k}-\left(\frac{\phi}{k_{g}}+\frac{1-\phi}{k_{r}}\right)}{\frac{1}{k_{g}}-\frac{1}{k_{r}}}\left(\alpha_{g}-\alpha_{r}\right) .
$$

This relation, which is general and does not depend on a specific model for the composite, recalls that the effective stiffness and thermal expansion of an isotropic linearly elastic two-phase composite are closely related. Consequently, thermal expansion results directly and consistently once a model has been chosen for stiffness, as stressed by Zaoui [21]. For instance, chosing the Reuss bound as an estimate for the elastic behavior nullifies the numerator in (6), and $\alpha=\phi \alpha_{g}+(1-\phi) \alpha_{r}$ results. This is equation (11) of [14], but $\alpha$ was rather fitted empirically to experimental results in the actual application of the model by Liu et al., which is not consistent with using the Reuss bound for the elastic behavior. In the present work, thermal expansion is deduced from the combination of (6) and (5) for the various models and bounds considered. Of course, $\alpha$ depends on $T$ through $\phi$ and $k$, which is a function of $\phi$, and possibly through $k_{r}$ also, like in the applications given below. Therefore, if the specimen were free to expand, its expansion along any direction would be

$$
\varepsilon^{t h}(T)=\int_{T_{0}}^{T} \alpha(T) \mathrm{d} T .
$$

when temperature changes from $T_{0}$ to $T$.

\subsection{Shape memory effect}

The shape memory effect requires a preliminary memory storage stage, defined for a negative increment $\mathrm{d} T$ at temperature $T$ between $T_{+}$and $T_{-}$, i.e. for a cooling step in the glass transition range. During this step, a fraction $\mathrm{d} \phi=\phi^{\prime}(T) \mathrm{d} T$ of polymer changes from rubbery to glassy, according to the basic 
assumption of the model. In this process, the relation between the average stress $\boldsymbol{\sigma}_{\mathrm{d} \phi}$ and average strain $\varepsilon_{\mathrm{d} \phi}$ in this infinitesimal volume fraction passes from $\boldsymbol{\sigma}_{\mathrm{d} \phi}=\boldsymbol{L}_{r}: \varepsilon_{\mathrm{d} \phi}$ to $\boldsymbol{\sigma}_{\mathrm{d} \phi}=\boldsymbol{L}_{g}:\left(\varepsilon_{\mathrm{d} \phi}-\varepsilon^{\star}\right)$. The latter expression combines a stiffness modification from $\boldsymbol{L}_{r}$ to $\boldsymbol{L}_{g}$ and the fact that the strain already reached in the rubbery state shifts to some extent the value for which the stress is zero in the glassy state. The strain shift $\varepsilon^{\star}=\left(\boldsymbol{L}_{r}^{-1}-\boldsymbol{L}_{g}^{-1}\right): \boldsymbol{\sigma}_{\mathrm{d} \phi}=\left(\boldsymbol{I}-\boldsymbol{L}_{g}^{-1}: \boldsymbol{L}_{r}\right): \varepsilon_{\mathrm{d} \phi}$ is due to the freezing of the part of the strain reached in the rubbery state that is related to chain mobility. In a finite strain context, it would correspond to a change in the reference configuration. This strain shift in the small volume fraction $\mathrm{d} \phi$ of rubbery phase, which can be described as a stress-free transformation strain that is similar to a thermal strain to some extent, also induces a strain shift $\mathrm{d} \varepsilon^{s}$ at the composite level, and the behavior of the polymer takes the form

$$
\boldsymbol{\sigma}=\boldsymbol{L}:\left(\varepsilon-\varepsilon^{t h}-\varepsilon^{s}\right) \quad \text { with } \quad \varepsilon^{s}=\int_{T_{+}}^{T} \mathrm{~d} \varepsilon^{s}
$$

when cooling proceeds, where $\boldsymbol{L}$ can be obtained from (3) and $\varepsilon^{t h}$ from (7). The $\varepsilon^{s}$ variable defines the strain storage that is at the root of the shape memory effect.

During a cooling increment, the applied strain may vary and the stress variation is obtained from (8) as follows:

$$
\mathrm{d} \boldsymbol{\sigma}=\boldsymbol{L}:\left(\mathrm{d} \boldsymbol{\varepsilon}-\mathrm{d} \varepsilon^{t h}-\mathrm{d} \varepsilon^{s}\right)+\left(\left.\frac{\partial \boldsymbol{L}}{\partial \phi}\right|_{T} \phi^{\prime}+\left.\frac{\partial \boldsymbol{L}}{\partial T}\right|_{\phi}\right): \boldsymbol{L}^{-1}: \boldsymbol{\sigma} \mathrm{d} T
$$

where two reasons for the variation of the stiffness tensor $\boldsymbol{L}$ of the polymer with temperature have been considered: the change in the volume fractions of the two constituents, which introduces a term proportional to $\phi^{\prime}$, and the effect of temperature on their stiffnesses. In the applications that follow, $L_{g}$ will not vary with temperature, but $L_{r}$ will be taken proportional to $T$, which is usual for rubber (Treloar [28], for instance) and was assumed by Liu et al. [14]. Considering that the shape memory effect is due to the phase change and, therefore, is associated with the $\phi^{\prime}$ term, (9) provides a way to compute the variation of the stored strain during a cooling increment, as

$$
\mathrm{d} \boldsymbol{\varepsilon}^{s}=\boldsymbol{L}^{-1}:\left.\frac{\partial \boldsymbol{L}}{\partial \phi}\right|_{T}: \boldsymbol{L}^{-1}: \boldsymbol{\sigma} \phi^{\prime} \mathrm{d} T=-\left.\frac{\partial \boldsymbol{L}^{-1}}{\partial \phi}\right|_{T}: \boldsymbol{\sigma} \phi^{\prime} \mathrm{d} T
$$

which shows that the stored strain increment depends on the current stress applied, whereas the thermal strain increment does not. Two special cases are worth of interest when (9) and (10) are combined, for a constant applied stress. First, assuming that both phases have constant stiffnesses, $\varepsilon=\varepsilon^{t h}$ is obtained, which means that the shape memory effect is not evident during cooling, with total strains being merely equal to thermal strains. If the applied stress is zero, this is the usual definition of thermal expansion, precisely. Second, disregarding thermal strains for clarity, $\mathrm{d} \varepsilon=\left.\frac{\partial \boldsymbol{L}^{-1}}{\partial T}\right|_{\phi}: \boldsymbol{\sigma} \mathrm{d} T$ is obtained, which implies that cooling $(\mathrm{d} T<0)$ induces an extension of a specimen under a uniaxial and constant tensile stress if $\boldsymbol{L}$ increases with $T$, which is consistent with $\boldsymbol{L}_{r}$ increasing with $T$. This corresponds to the Gough-Joule effect, which is commonly observed in rubber (Treloar [28], for instance). This effect is maximal when $\phi=0\left(\boldsymbol{L}=\boldsymbol{L}_{r}\right)$, decreases when cooling proceeds, and vanishes when $\phi=1\left(\boldsymbol{L}=\boldsymbol{L}_{g}\right)$.

If a uniform stress model is used, $\boldsymbol{L}^{-1}=\phi \boldsymbol{L}_{g}^{-1}+(1-\phi) \boldsymbol{L}_{r}^{-1}$ applies, and the rightmost expression in (10) gives immediately

$$
\mathrm{d} \varepsilon^{s}=\left(\boldsymbol{L}_{r}^{-1}-\boldsymbol{L}_{g}^{-1}\right): \boldsymbol{\sigma} \phi^{\prime} \mathrm{d} T
$$

which is is in agreement with equation (22) of [17] but not with equation (30) of [14], where the $\boldsymbol{L}_{g}^{-1}$ term is missing. This omission implies that there is a shape memory effect in the Liu et al. [14] approach when both phases have the same moduli, whereas (11) leads to $\mathrm{d} \boldsymbol{\varepsilon}^{s}=\mathbf{0}$. The difference between the two formulations is negligible, nevertheless, in such practical cases as considered in [14], where $\boldsymbol{L}_{r}^{-1} \gg \boldsymbol{L}_{g}^{-1}$. 
Up to this point, cooling steps only have been considered, during which the glassy volume fraction increases, and the process of storing the shape memory has been described. How this memory is recalled during heating steps that are performed between the $T_{-}$and $T_{+}$temperatures consists simply in substracting from the current value $\varepsilon^{s}$ the same $\mathrm{d} \varepsilon^{s}$ increment that was added when the $\mathrm{d} \phi$ volume fraction concerned passed from rubbery to glassy. In other words, (9) is applied with $\mathrm{d} \varepsilon^{s}$ not given by (10) but read from the cooling history. This "unrolls" the history of the stored strain and ensures that no hysteresis is generated in the shape memory process with, therefore, a strain $\varepsilon=-\varepsilon^{s}$ being induced in addition to thermal strains when heating under $\boldsymbol{\sigma}=\mathbf{0}$ conditions, for instance, which is typical of a shape memory effect. This also implies that the $\varepsilon^{s}$ history must be recorded. More precisely, it is sufficient to store $\mathrm{d} \varepsilon^{s} / \mathrm{d} T$ for the "net cooling history" as defined by Chen and Lagoudas [16], i.e. for negative temperature increments that have not been compensated yet by positive increments between $T_{+}$and the current temperature. In the finite element simulation of general three-dimensional loadings, this involves the storage of the 6 independent components of a symmetric second-order tensor at a series of temperature values in the transition range and at each integration point.

When a uniaxial stress $\sigma$ is applied to a specimen with all mechanical properties assumed isotropic, the variation of the component of the stored strain that is parallel to the applied stress is obtained from (10) as

$$
\mathrm{d} \varepsilon^{s}=\left(\left.\frac{1}{9 k^{2}} \frac{\partial k}{\partial \phi}\right|_{T}+\left.\frac{1}{3 \mu^{2}} \frac{\partial \mu}{\partial \phi}\right|_{T}\right) \sigma \phi^{\prime} \mathrm{d} T
$$

during cooling. The two partial derivatives have trivial expressions for the Reuss and Voigt bounds, and take the following forms if the Hashin and Shtrikman bounds are used:

$$
\left.\frac{\partial k}{\partial \phi}\right|_{T}=\frac{k-k_{r}}{\phi} \frac{k^{\star}+k}{k^{\star}+k_{r}} \quad \text { and }\left.\quad \frac{\partial \mu}{\partial \phi}\right|_{T}=\frac{\mu-\mu_{r}}{\phi} \frac{\mu^{\star}+\mu}{\mu^{\star}+\mu_{r}}
$$

with, when $\phi=0$ :

$$
\left.\frac{\partial k}{\partial \phi}\right|_{T}=\left(k_{g}-k_{r}\right) \frac{k^{\star}+k_{r}}{k^{\star}+k_{g}} . \quad \text { and }\left.\quad \frac{\partial \mu}{\partial \phi}\right|_{T}=\left(\mu_{g}-\mu_{r}\right) \frac{\mu^{\star}+\mu_{r}}{\mu^{\star}+\mu_{g}} .
$$

The coupled equations of the self-consistent model lead to a linear system with respect to $\partial k /\left.\partial \phi\right|_{T}$ and $\partial \mu /\left.\partial \phi\right|_{T}$ defined by:

and

$$
\left.\frac{\partial k}{\partial \phi}\right|_{T}=\frac{k-k_{r}}{\phi} \frac{k^{\star}+k}{k^{\star}+k_{r}}+\left.\frac{4}{3} \frac{1-\phi}{\phi}\left(\frac{k-k_{r}}{k^{\star}+k_{r}}\right)^{2} \frac{\partial \mu}{\partial \phi}\right|_{T}
$$

$$
\begin{aligned}
\left.\frac{\partial \mu}{\partial \phi}\right|_{T}=\frac{\mu-\mu_{r}}{\phi} \frac{\mu^{\star}+\mu}{\mu^{\star}+\mu_{r}} & \\
+ & \frac{8}{3} \frac{1-\phi}{\phi}\left(\frac{\mu-\mu_{r}}{\mu^{\star}+\mu_{r}}\right)^{2} \frac{\mu^{2}}{(k+2 \mu)^{2}}\left[\left.\frac{5}{8} \frac{\partial k}{\partial \phi}\right|_{T}+\left.\left(1+\frac{k}{\mu}+\frac{9}{16} \frac{k^{2}}{\mu^{2}}\right) \frac{\partial \mu}{\partial \phi}\right|_{T}\right]
\end{aligned}
$$

with (14) still applying when $\phi=0$. This system can be solved analytically, or rather numerically with a fixed-point method.

The relation between increments of uniaxial stress, temperature and total strain (in the direction of the applied stress) is obtained from (9) as:

$$
\begin{aligned}
& \mathrm{d} \sigma=E\left[\mathrm{~d} \varepsilon-\alpha \mathrm{d} T-\mathrm{d} \varepsilon^{s}+\left(\left.\frac{1}{9 k^{2}} \frac{\partial k}{\partial \phi}\right|_{T}+\left.\frac{1}{3 \mu^{2}} \frac{\partial \mu}{\partial \phi}\right|_{T}\right) \sigma \phi^{\prime} \mathrm{d} T+\right. \\
&\left.+\left(\left.\frac{1}{9 k^{2}} \frac{\partial k}{\partial T}\right|_{\phi}+\left.\frac{1}{3 \mu^{2}} \frac{\partial \mu}{\partial T}\right|_{\phi}\right) \sigma \mathrm{d} T\right]
\end{aligned}
$$


where $E=9 k \mu /(3 k+\mu)$ denotes the Young's modulus and $\alpha$ is given by (6). Assuming that $k_{r}$ and $\mu_{r}$ are proportional to $T$, as mentioned above, leads to the following simple expressions

$$
\left.\frac{1}{9 k^{2}} \frac{\partial k}{\partial T}\right|_{\phi}+\left.\frac{1}{3 \mu^{2}} \frac{\partial \mu}{\partial T}\right|_{\phi}=\frac{1-\phi}{T}\left(\frac{1}{9 k_{r}}+\frac{1}{3 \mu_{r}}\right)
$$

and

$$
\left.\frac{1}{9 k^{2}} \frac{\partial k}{\partial T}\right|_{\phi}+\left.\frac{1}{3 \mu^{2}} \frac{\partial \mu}{\partial T}\right|_{\phi}=\frac{1-\phi}{T}\left(\frac{k_{r}}{9 k^{2}}+\frac{\mu_{r}}{3 \mu^{2}}\right)
$$

when the Reuss and Voigt bounds are used, respectively, to evaluate $k$ and $\mu$. The corresponding expressions for the Hashin and Shtrikman bounds are more involved:

$$
\begin{aligned}
\left.\frac{1}{9 k^{2}} \frac{\partial k}{\partial T}\right|_{\phi}+ & \left.\frac{1}{3 \mu^{2}} \frac{\partial \mu}{\partial T}\right|_{\phi}=\frac{1}{T(1-\phi)} \frac{k_{r}}{9 k^{2}}\left(\frac{k-k_{g}}{k_{r}-k_{g}}\right)^{2}\left[1+\frac{4 \phi}{3}\left(\frac{k_{r}-k_{g}}{k^{\star}+k_{g}}\right) \frac{\mu_{r}}{k_{r}}\right]+ \\
& +\frac{1}{T(1-\phi)} \frac{\mu_{r}}{3 \mu^{2}}\left(\frac{\mu-\mu_{g}}{\mu_{r}-\mu_{g}}\right)^{2}\left[1+\frac{8 \phi}{3}\left(\frac{\mu_{r}-\mu_{g}}{\mu^{\star}+\mu_{g}}\right) \frac{\mu_{r}^{2}}{\left(k_{r}+2 \mu_{r}\right)^{2}}\left(1+\frac{13}{8} \frac{k_{r}}{\mu_{r}}+\frac{9}{16} \frac{k_{r}^{2}}{\mu_{r}^{2}}\right)\right]
\end{aligned}
$$

and

$$
\left.\frac{1}{9 k^{2}} \frac{\partial k}{\partial T}\right|_{\phi}+\left.\frac{1}{3 \mu^{2}} \frac{\partial \mu}{\partial T}\right|_{\phi}=\frac{1}{T(1-\phi)}\left[\frac{k_{r}}{9 k^{2}}\left(\frac{k-k_{g}}{k_{r}-k_{g}}\right)^{2}+\frac{\mu_{r}}{3 \mu^{2}}\left(\frac{\mu-\mu_{g}}{\mu_{r}-\mu_{g}}\right)^{2}\right]
$$

for the lower and upper bounds, respectively, with the right-hand terms being equal to zero when $\phi=1$. The coupled equations of the self-consistent model lead to two linear systems to be solved (a fixed-point method is convenient). One, with respect to $\partial k /\left.\partial k_{r}\right|_{T}$ and $\partial \mu /\left.\partial k_{r}\right|_{T}$, is defined by:

$$
\left.\frac{\partial k}{\partial k_{r}}\right|_{T}=\frac{1}{1-\phi}\left(\frac{k-k_{g}}{k_{r}-k_{g}}\right)^{2}+\left.\frac{4}{3} \frac{\phi}{1-\phi}\left(\frac{k-k_{g}}{k^{\star}+k_{g}}\right)^{2} \frac{\partial \mu}{\partial k_{r}}\right|_{T}
$$

and

$$
\left.\frac{\partial \mu}{\partial k_{r}}\right|_{T}=\frac{8}{3} \frac{\phi}{1-\phi}\left(\frac{\mu-\mu_{g}}{\mu^{\star}+\mu_{g}}\right)^{2} \frac{\mu^{2}}{(k+2 \mu)^{2}}\left[\left.\frac{5}{8} \frac{\partial k}{\partial k_{r}}\right|_{T}+\left.\left(1+\frac{k}{\mu}+\frac{9}{16} \frac{k^{2}}{\mu^{2}}\right) \frac{\partial \mu}{\partial k_{r}}\right|_{T}\right]
$$

the other, with respect to $\partial k /\left.\partial \mu_{r}\right|_{T}$ and $\partial \mu /\left.\partial \mu_{r}\right|_{T}$, is defined by:

$$
\left.\frac{\partial k}{\partial \mu_{r}}\right|_{T}=\left.\frac{4}{3} \frac{\phi}{1-\phi}\left(\frac{k-k_{g}}{k^{\star}+k_{g}}\right)^{2} \frac{\partial \mu}{\partial \mu_{r}}\right|_{T}
$$

and

$$
\begin{aligned}
\left.\frac{\partial \mu}{\partial \mu_{r}}\right|_{T}=\frac{1}{1-\phi}\left(\frac{\mu-\mu_{g}}{\mu_{r}-\mu_{g}}\right)^{2}+ & \\
& \quad+\frac{8}{3} \frac{\phi}{1-\phi}\left(\frac{\mu-\mu_{g}}{\mu^{\star}+\mu_{g}}\right)^{2} \frac{\mu^{2}}{(k+2 \mu)^{2}}\left[\left.\frac{5}{8} \frac{\partial k}{\partial \mu_{r}}\right|_{T}+\left.\left(1+\frac{k}{\mu}+\frac{9}{16} \frac{k^{2}}{\mu^{2}}\right) \frac{\partial \mu}{\partial \mu_{r}}\right|_{T}\right] .
\end{aligned}
$$

Finally, the four partial derivatives given by these systems are introduced into

$$
\left.\frac{1}{9 k^{2}} \frac{\partial k}{\partial T}\right|_{\phi}+\left.\frac{1}{3 \mu^{2}} \frac{\partial \mu}{\partial T}\right|_{\phi}=\frac{1}{9 k^{2}}\left(\left.\frac{k_{r}}{T} \frac{\partial k}{\partial k_{r}}\right|_{T}+\left.\frac{\mu_{r}}{T} \frac{\partial k}{\partial \mu_{r}}\right|_{T}\right)+\frac{1}{3 \mu^{2}}\left(\left.\frac{k_{r}}{T} \frac{\partial \mu}{\partial k_{r}}\right|_{T}+\left.\frac{\mu_{r}}{T} \frac{\partial \mu}{\partial \mu_{r}}\right|_{T}\right)
$$

with the right-hand term equal to zero when $\phi=1$. 


\section{Comparison with experimental results}

The specimens were loaded uniaxially in the experiments described by Liu et al. [14], and the equations given at the end of the previous section can therefore be used. The coefficients of thermal expansion were measured as $\alpha_{g}=0.9 \times 10^{-4} / \mathrm{K}$ and $\alpha_{r}=1.8 \times 10^{-4} / \mathrm{K}$. The bulk and shear moduli of the two phases are required in the present formulation, but only the Young's moduli are given in [14], with a linear variation with respect to temperature for the rubbery phase: $E_{g}=750 \mathrm{MPa}$ and $E_{r}=8.8(T / 358) \mathrm{MPa}$, with $T$ in kelvins. The Poisson's ratios were not measured, but a value of 0.4 can reasonably be taken for the glassy phase. For the rubbery phase, a value of 0.498 leads to a drop of the shear modulus by about two decades through the glass transition, and a drop by a factor of 1.7 for the bulk modulus, which are acceptable orders of magnitude.

In the tests, the material is first heated, stress free, at a temperature $T_{h}=358 \mathrm{~K}$, above the glass transition, where it is assumed to be completely rubbery $(\phi=0)$. This state is taken as the reference for strains and, therefore, $T_{0}=T_{h}$ is used to compute thermal strains. Tensile, compressive, or zero stresses are then applied at temperature $T_{h}$, which induce a strain $\varepsilon_{0}$ of $9.1 \%,-9.1 \%$ or 0 , that is kept constant in the next step during which the temperature is lowered to $T_{l}=273 \mathrm{~K}$. At the latter temperature, the material is glassy $(\phi=1)$ and the specimen is unloaded, with stresses being relaxed. In the last step, the specimen is heated back to $T_{h}$ with a length that is either fixed (stress recovery test, stresses are measured) or free to vary (strain recovery test, strains are measured). With the 3 values used for $\varepsilon_{0}$, this leads to a set of 6 different histories.

The same procedure as in [14] is followed, where the $\phi(T)$ function is fitted on the strain recovery that results from a $\varepsilon_{0}=-9.1 \%$ compression at high temperature. In order to reduce the number of parameters, $T_{+}=T_{h}$ and $T_{-}=T_{l}$ are used, like in [14], which leaves the exponents $m$ and $n$ to be determined. The effect of modifying $T_{+}$and $T_{-}$, with a narrower temperature transition range, is left for future work. The least square minimization used the full set of equations of the model, since strain recovery involves the polymer elastic properties and thermal expansion and the shape memory effect, which was integrated numerically with very small temperature steps. A good fit of the experimental points could be obtained in this way, as shown by the RMS values given in Table 1 that can be compared with the average strain observed during the test, which was $-7.54 \times 10^{-2}$. It may be noted that the best fit is obtained with the Voigt and Hashin-Shtrikman upper bounds while the self-consistent model gives the worst agreement. Except for the latter, with a slightly more apparent difference, all models lead to very similar curves as in Figure 1, where the optimized Voigt model has been used. This figure also shows that the model gives a good prediction for the strain recovery test performed after a tension of $\varepsilon_{0}=9.1 \%$ at high temperature. This also applies to the optimized Reuss model and Hashin and Shtrikman bounds, with a larger discrepancy for the self-consistent model again. The central curve in Figure 1 pertains to $\varepsilon_{0}=0$ and, consequently, to a very small memory effect, about twice smaller than thermal strains, with all models giving good predictions in this non discriminant case. Using the coefficients given in Table 1 , Figure 2 shows the significant differences between the optimized $\phi(T)$ functions obtained for the various models, which are compensated by large differences in the models themselves to give similar predictions in Figure 1. This compensation will play differently for other loadings, and the predictions of the models will differentiate clearly, as shown below. The general trend in Figure 2 is quite intuitive: to get similar responses at a given temperature, stiffer models (i.e. models using stiffer comparison materials) need smaller volume fractions $\phi$ of stiff (glassy) phase.

A good agreement with the three experimental strain recovery plots was also obtained by Liu et al. [14] and by Chen and Lagoudas [17], but using additional data, since the $\alpha(T)$ thermal expansion coefficient was fitted, with Chen and Lagoudas [17] furthermore using a fitted stress recovery curve. In the present approach, $\alpha(T)$ is computed from the $\phi(T)$ function for each composite model, as given by (6), and it is 
Table 1

\begin{tabular}{cccccc} 
& \multicolumn{6}{c}{ Reuss HS lower Self cons. HS upper Voigt } \\
\hline$m$ & 13.31 & 10.55 & 4.57 & 3.46 & 2.77 \\
$n$ & 7.77 & 7.69 & 2.91 & 5.26 & 4.94 \\
RMS $\left(\times 10^{3}\right)$ & 1.59 & 1.59 & 2.28 & 1.24 & 1.23
\end{tabular}

Optimized exponents $m$ and $n$ for the $\phi(T)$ function (2) to fit the experimental strain recovery observed by Liu et al. [14] after a compression of $-9.1 \%$. RMS is the root mean square difference between the curve and the experimental points.

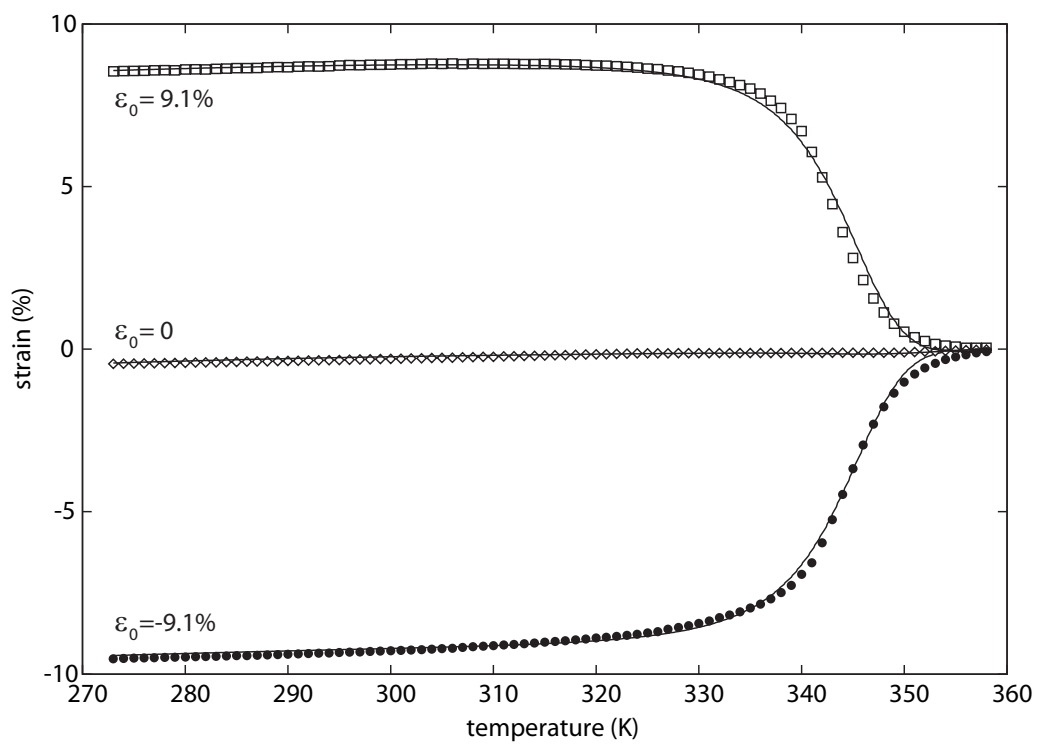

Figure 1. Comparison between the Voigt (uniform strain) variant of the model (lines) and the experimental results obtained by Liu et al. [14] for strain recovery after various prestrains applied at high temperature (circles: $\varepsilon_{0}=-9.1 \%$, diamonds: $\varepsilon_{0}=0$, squares: $\left.\varepsilon_{0}=9.1 \%\right)$. Only the data shown in full symbols have been used to fit the $\phi(T)$ function.

interesting to compare the corresponding predictions of thermal strains with the experimental results of [14]. The average between the cooling and heating experiments on a stress-free specimen, which lead to very close results, is shown in Figure 3. The $m$ and $n$ values given in Table 1 have been used to apply the model variants and it appears immediately that the agreement with experiments is much better for the Voigt bound and the Hashin and Shtrikman upper bound. Therefore, although the Reuss model and the Hashin and Shtrikman lower bound could provide a good fit and a good prediction for strain recoveries after compression and tension, respectively, these variants must be eliminated when predictions of thermal expansion are considered. The optimized function obtained for the Reuss model was found close to the $\phi(T)$ function used by Liu et al. [14], as could be observed in Figure 2, and therefore the latter function leads also to an unsatisfactory prediction of thermal strains when applied to (6) with a Reuss model, as shown in Figure 3. The coupling between elastic behavior and thermal expansion was disregarded by Liu et al. [14], who rather used a fitted $\alpha(T)$ function in the actual application of their model, as stated in Table 1 of [14]. It may be noted incidentally that this function does not recover the $\alpha_{g}=0.9 \times 10^{-4} / \mathrm{K}$ and $\alpha_{r}=1.8 \times 10^{-4} / \mathrm{K}$ experimental values for glassy and rubbery states, but rather $\alpha_{g}=0.72 \times 10^{-4} / \mathrm{K}$ and $\alpha_{r}=1.92 \times 10^{-4} / \mathrm{K}$.

The model can now be applied to stress recovery tests, and Figure 4 compares the predictions of the Voigt variant with the experimental results of [14]. The variant using the upper Hashin and Shtrikman bound leads to very similar curves. The global agreement between theory and experiment compares well 


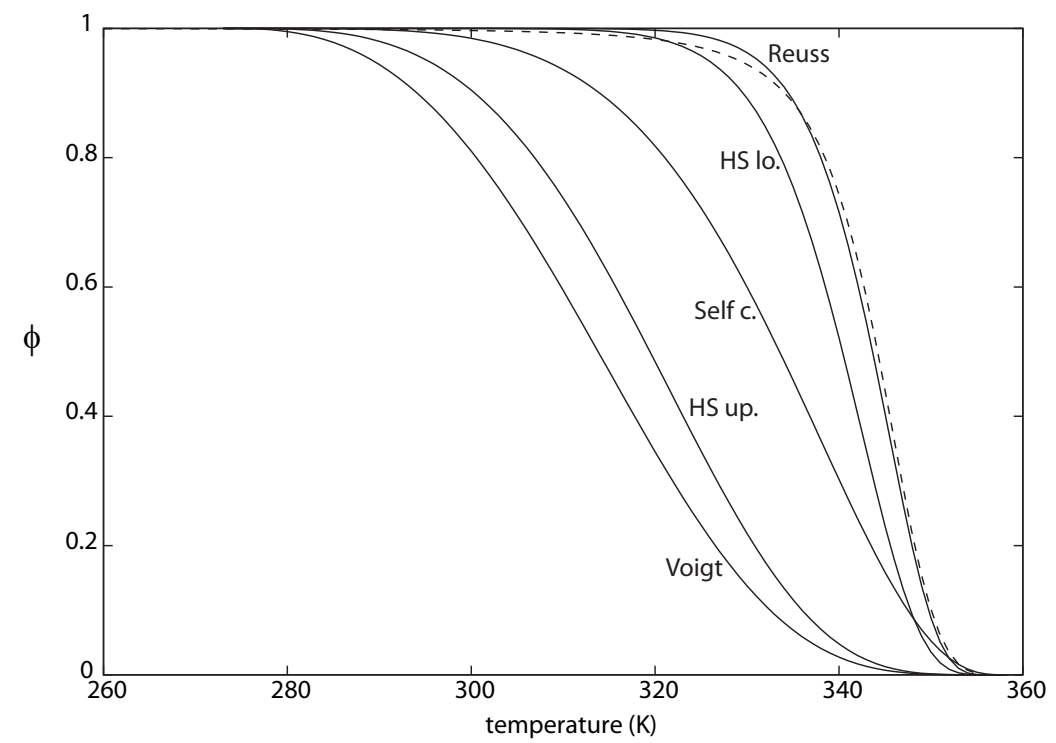

Figure 2. Optimized $\phi(T)$ functions obtained for the various models considered (Reuss and Voigt bounds, Hashin and Shtrikman lower and upper bounds, self-consistent model). The $\phi(T)$ function used by Liu et al. [14] is also shown (dashed line).

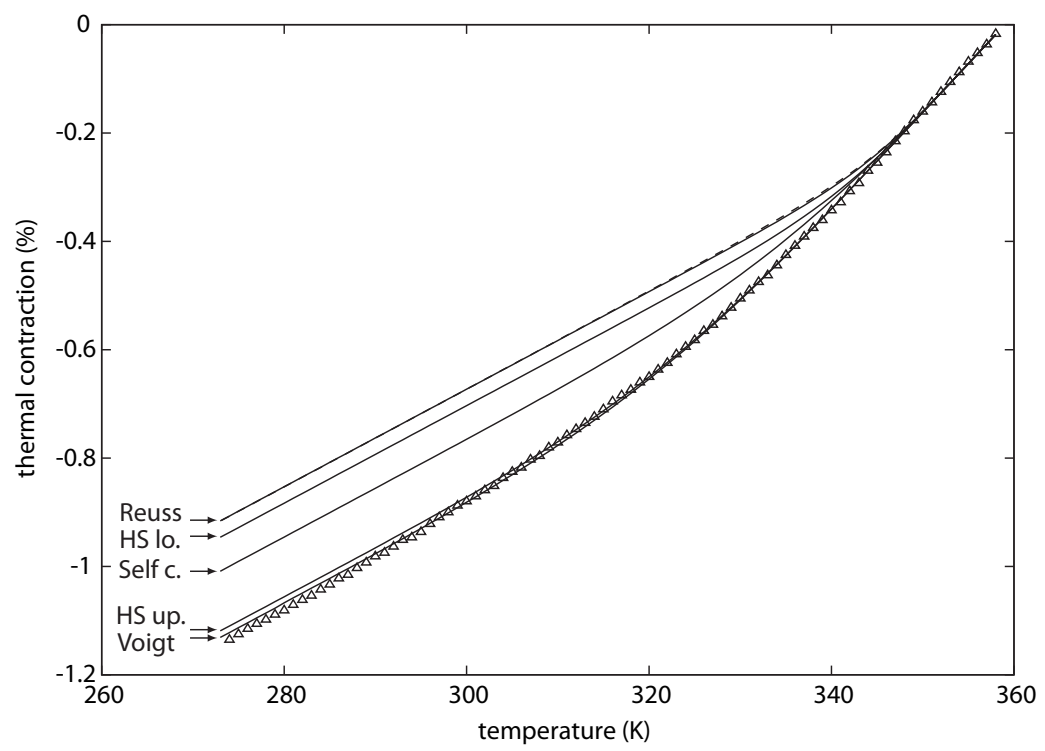

Figure 3. Predicted thermal strain when each model variant is considered with its own $\phi(T)$ function optimized on a strain recovery test. Comparison with the experimental results (symbols) obtained by Liu et al. [14]. The dashed curve is the combination of the Reuss model for thermal expansion with the $\phi(T)$ function used by Liu et al. [14].

with what could be obtained by Liu et al. [14] and Chen and Lagoudas [17]. Like in these previous works, no hysteresis is produced when heating is applied immediately after cooling, keeping boundary conditions unchanged. This means that the three upper curves in Figure 4 apply for cooling as well as for immediate subsequent heating, whereas experimental data reveal a slight hysteresis that produces two series of points 


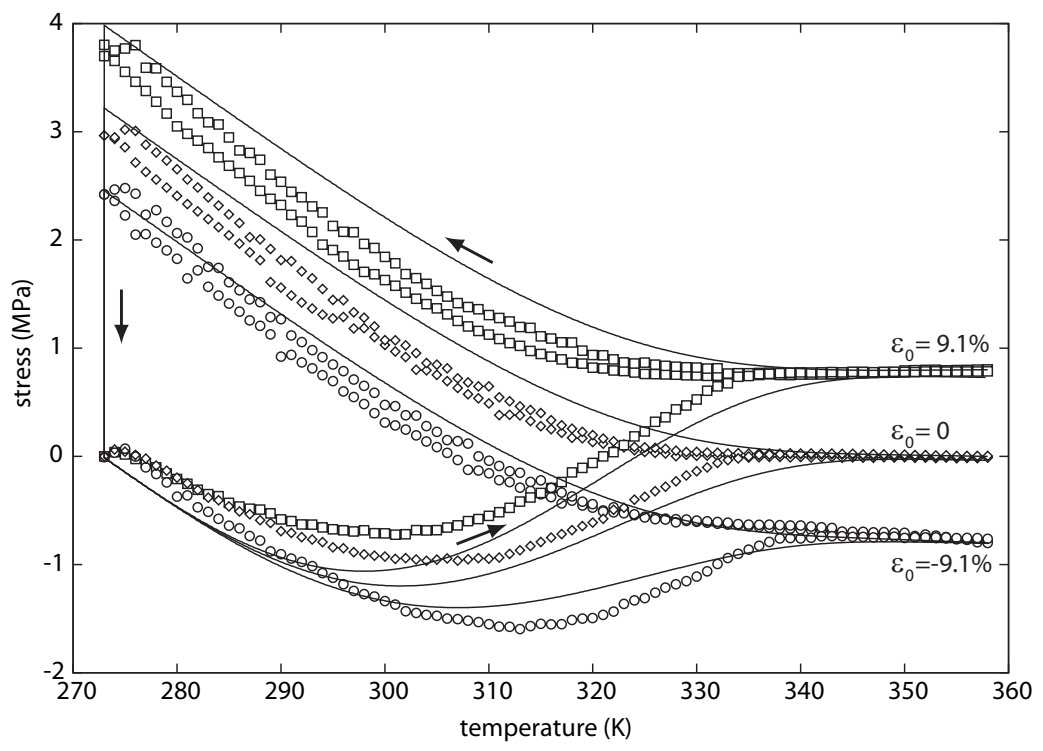

Figure 4. Comparison between the Voigt (uniform strain) variant of the model (lines) and the experimental results obtained by Liu et al. [14] for cooling and stress recovery after various prestrains applied at high temperature (circles: $\varepsilon_{0}=-9.1 \%$, diamonds: $\varepsilon_{0}=0$, squares: $\varepsilon_{0}=9.1 \%$ ).

in each case. After stress unloading, heating induces a stress recovery that is reasonably well predicted by the model (lower curves in Figure 4), and it may be recalled that a single (strain recovery) curve has been used for fitting so far. One difference between theory and experiment is an opposite trend when heating begins: the experimental curves show an increasing stress, when the model predicts a decrease. The same difference with experiments was also found by Liu et al. [14] and Chen and Lagoudas [17]. Actually, the model prediction corresponds to the slope at the lowest temperature being trivially equal to $\alpha_{g} E_{g}$, since the material is glassy, with this result applying for both the upper and lower curves in Figure 4 . Therefore, the different trend obtained in the lower series of experimental points may be questioned. Figure 5 shows the predictions of the Reuss variant of the model, which are clearly poor, although the results were correct for strain recovery tests. This is consistent with the unsatisfactory prediction of the $\alpha(T)$ function already discussed about Figure 3 and stresses that a good fit on a strain recovery curve, with a good prediction on another, is not enough for a model to predict correctly the shape memory effect under various conditions.

In order to understand the role of the shape memory effect on stress recovery, Figure 6 shows the results given by the Voigt variant of the model when the shape memory effect is turned off (by taking $\mathrm{d} \varepsilon^{s}=\mathbf{0}$ ) in the last heating stage, still using the same optimized $\phi$ function. The three lower curves of Figure 4 are also shown for comparison. It is noted first that the effect of prestrain disappears, since it is stored in the $\varepsilon^{s}$ variable that is inactive now. It is also observed that the general conformation of the stress recovery curves is maintained when the shape memory is turned off. Therefore, this conformation is largely due to thermal strains and to the variations of the properties of the composite with temperature, with shape memory modulating the amplitude of the curves. The stress at the end of the recovery process is slightly lower than the value $\left(E_{r} \varepsilon_{0}\right)$ that has been prescribed initially at high temperature, but this was not visible in Figure 4 because of the size of the experimental points. This effect is clearly evident for the $\varepsilon_{0}=0$ curve in Figure 6 because the reference is 0 , but it does exist also for the two other dashed curves. Its value is $-E_{r} / E_{g}$ times the peak stress reached at the end of the cooling stage with fixed strain, which is relaxed before stress recovery starts, plus an extra term when shape memory is turned off. This can be obtained easily by noting that a thermal cycle from and back to high temperature leads to $\varepsilon^{\text {th }}=\mathbf{0}$ 


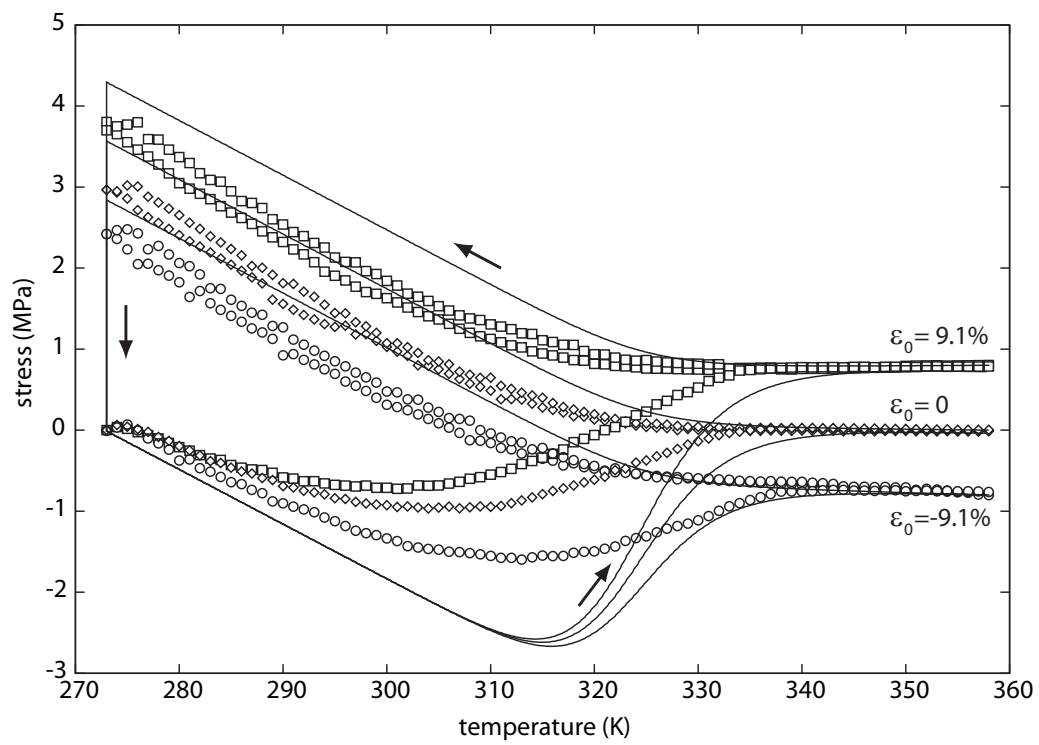

Figure 5. Comparison between the Reuss (uniform stress) variant of the model (lines) and the experimental results obtained by Liu et al. [14] for cooling and stress recovery after various prestrains applied at high temperature (circles: $\varepsilon_{0}=-9.1 \%$, diamonds: $\varepsilon_{0}=0$, squares: $\varepsilon_{0}=9.1 \%$ ).

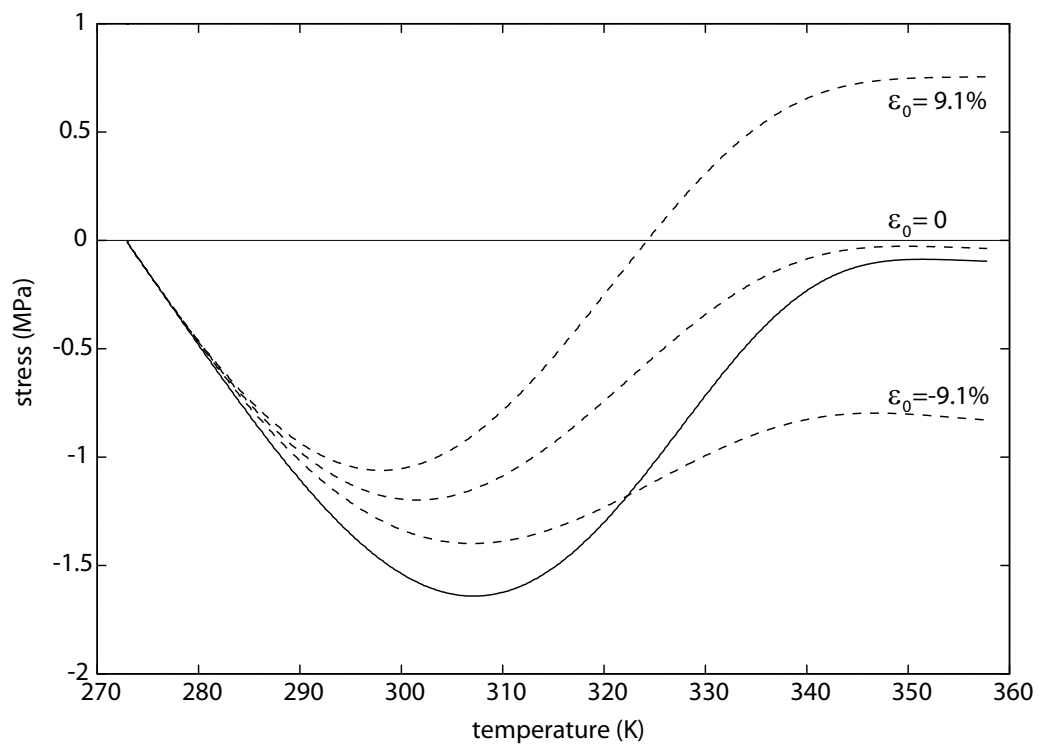

Figure 6. Same lower curves as in Figure 4 (dashed lines), compared with the result obtained by turning the shape memory off during stress recovery (unbroken line).

and $\varepsilon^{s}=\mathbf{0}$. The same argument explains why there is no residual strain in the strain recovery curves in Figure 1.

It may be mentioned finally that neglecting the Gough-Joule effect in (9) by disregarding the partial derivative of $\boldsymbol{L}$ with respect to $T$ in order to simplify the model, but still keeping $\boldsymbol{L}_{r}$ to vary with $T$ in the computation of $\boldsymbol{L}$, has some influence on the strain recovery results. Using the suitably optimized $\phi$ 
function, this induces a small residual strain after the specimen is back to the highest temperature, with a sign opposite to the prestrain. In other words, this simplification would lead to a crossing of the upper and lower curves in Figure 1 at high temperatures. In contrast, the effect on the stress recovery curves is less evident and the general aspect of the curves is maintained.

\section{Concluding remarks}

This study has addressed one point that was left open by Chen and Lagoudas [16] about combining the model of Liu et al. [14] with homogenization models that would be more accurate than the uniform stress assumption. This has been performed in the limited framework of linear small-strain elasticity. Using a result of the theory of composites that relates thermal expansion and elastic properties of a two-phase material has allowed to get a model that predicts reasonably well a series of strain recovery tests performed by Liu et al. [14] on a shape memory polymer, a series of stress recovery tests, and the thermal expansion that has been measured experimentally. All these results are obtained by using a single experimental curve to fit a function defined by two parameters.

The results are at least as good as those obtained in previous studies that disregarded the connection between thermal expansion and elastic properties and that used either two or three fitted experimental curves. These works were based on a uniform stress assumption, but the approach developed here shows that a uniform strain hypothesis does lead to good predictions, whereas a consistent use of the uniform stress hypothesis to predict both the elastic properties and thermal expansion gives poor results. In addition, more elaborate models, like the Hashin and Shtrikman bounds (or the Mori and Tanaka model, equivalently) or the self-consistent model, either do not improve significantly over the uniform stress model or are close to the uniform strain approach. The Mori and Tanaka model where rubbery particles are randomly distributed in a glassy matrix is found close to the uniform strain model, which suggests that an increasingly dense continuous network of glassy phase develops during cooling through the glass transition. This contrasts with the usual assumption of glassy particles growing in a continuous rubbery phase, as illustrated in Figure 9 of [14] and as considered in the version of the Mori and Tanaka model used by Wang et al. [18]. It may be noted finally that a uniform strain was also assumed in the model of Qi et al. [9] but in the quite different context of a viscoelastic two-phase mixture.

The present approach is quite modular. Various composite models have been implemented and more can be considered easily. The type of $\phi(T)$ function, which defines the change in the volume fractions of rubbery and glassy phases, can be modified conveniently, and the experimental reference curve used to fit $\phi(T)$ can also be varied. The model restricts to small deformations, but extension to finite strain can be contemplated now by adapting the Chen and Lagoudas [16] approach to the uniform strain hypothesis that has been shown here to be appropriate.

\section{Acknowledgements}

PG is very grateful to André Zaoui for the amount of knowledge imparted in the homogenization techniques and for friendly stimulating encouragements. This work was supported by the French National Research Agency through project REFORM 10-JCJC-0917.

\section{References}

[1] C. Liu, H. Qin, P.T. Mather, Review of progress in shape-memory polymers. J. Mater. Chem. 17 (2007) 1543-1558. 
[2] I.A. Rousseau, Challenges of shape memory polymers: a review of the progress toward overcoming SMPS's limitations. Polym. Eng. Sci. 48 (2008) 2075-2287.

[3] P.T. Mather, X. Luo, I.A. Rousseau, Shape memory polymer research. Annu. Rev. Mater. Res. 39 (2009) $445-471$.

[4] H. Tobushi, T. Hashimoto, S. Hayashi, E. Yamada, Thermomechanical constitutive modeling in shape memory polymer of polyurethane series. J. Intell. Mater. Syst. Struct. 8 (1997) 711-718.

[5] A. Bhattacharyya, H. Tobushi, Analysis of the isothermal mechanical response of a shape memory polymer rheological model. Polym. Eng. Sci. 40 (2000) 2498-2510.

[6] H. Tobushi, K. Okumura, S. Hayashi, N. Ito, Thermomechanical constitutive model of shape memory polymer. Mech. Mater. 33 (2001) 545-554.

[7] J.R. Lin, L.W. Chen, Shape-memorized crosslinked ester-type polyurethane and its mechanical viscoelastic model. J. Appl. Polym. Sci. 73 (1999) 1305-1319.

[8] V. Kafka, Shape memory polymers: a mesoscale model of the internal mechanism leading to the SM phenomena. Int. J. Plast. 24 (2008) 1533-1548.

[9] H.J. Qi, T.D. Nguyen, F. Castro, C.M. Yakacki, R. Shandas, Finite deformation thermo-mechanical behavior of thermally induced shape memory polymers. J. Mech. Phys. Solids 56 (2008) 1730-1751.

[10] T.D. Nguyen, H.J. Qi, F. Castro, K.N. Long, A thermoviscoelastic model for amorphous shape memory polymers: incorporating structural and stress relaxation. J. Mech. Phys. Solids 56 (2008) 2792-2814.

[11] V. Srivastava, S.A. Chester, L. Anand, Thermally actuated shape-memory polymers: experiments, theory, and numerical simulations. J. Mech. Phys. Solids 58 (2010) 1100-1124.

[12] G. Barot, I.J. Rao, I.J., Constitutive modeling of the mechanics associated with crystallizable shape memory polymers. Z. angew. Math. Phys. 57 (2006) 652-681.

[13] G. Barot, I.J. Rao, K.R. Rajagopal, A thermodynamic framework for the modeling of crystallizable shape memory polymers. Int. J. Eng. Sci. 46 (2008) 325-351.

[14] Y. Liu, K. Gall, M.L. Dunn, A.R. Greenberg, J. Diani, J., Thermomechanics of shape memory polymers: uniaxial experiments and constitutive modeling. Int. J. Plast. 22 (2006) 279-313.

[15] J. Diani, Y. Liu, K. Gall, Finite strain 3D thermoviscoelastic constitutive model for shape memory polymers. Polym. Eng. Sci., 46 (2006) 484-492.

[16] Y.C. Chen, D.C. Lagoudas, A constitutive theory for shape memory polymers. Part I: Large deformations. J. Mech. Phys. Solids 56 (2008) 1752-1765.

[17] Y.C. Chen, D.C. Lagoudas, A constitutive theory for shape memory polymers. Part II: A linearized model for small deformations. J. Mech. Phys. Solids, 56 (2008) 1766-1778.

[18] Z.D. Wang, D.F. Li, Z.Y. Xiong, R.N. Chang, Modeling thermomechanical behaviors of shape memory polymer. J. Appl. Polym. Sci. 113 (2009) 651-656.

[19] S. Reese, M. Böl, D. Christ, Finite element-based multi-phase modelling of shape memory polymer stents. Comput. Methods Appl. Mech. Eng. 199 (2010) 1276-1286.

[20] J.R. Willis, The overall elastic response of composite materials. ASME J. Appl. Mech. 50 (1983) $1202-1209$.

[21] A. Zaoui, Continuum micromechanics: a survey. J. Engng Mech. 128 (2002) 808-816.

[22] R. Hill, A self-consistent mechanics of composite materials. J. Mech. Phys. Solids 13 (1965) 213-222.

[23] Z. Hashin, S. Shtrikman, A variational approach to the theory of the elastic behaviour of multiphase materials. J. Mech. Phys. Solids 11 (1963) 127-140.

[24] T. Mori, K. Tanaka, Average stress in matrix and average elastic energy of materials with misfitting inclusions. Acta Mater. 21 (1973) 571-574.

[25] Y. Benveniste, A new approach to the application of Mori-Tanaka's theory in composite materials. Mech. Mater. 6 (1987) 147-157.

[26] V.M. Levin, Thermal expansion coefficients of hererogeneous materials. Mekhanika Tvergodo Tela 2 (1967) 88-94.

[27] B.W. Rosen, Z. Hashin, Effective thermal expansion coefficients and specific heats of composite materials. Int. J. Eng. Sci. 8 (1970) 157-173.

[28] L.R.G. Treloar, The Physics of Rubber Elasticity, Oxford University Press, 1980. 